\title{
X-ray Diffraction Analysis to Clarify the Unusual Origin of an Intraocular Foreign Body
}

\author{
Francisco J. Ascaso ${ }^{1,2}$ and Javier Blasco ${ }^{3^{*}}$ \\ ${ }^{1}$ Department of Ophthalmology, 'Lozano Blesa” University Clinic Hospital, Zaragoza, Spain. \\ ${ }^{2}$ Aragon Health Sciences Institute, Zaragoza, Spain. \\ ${ }^{3}$ Instituto de Ciencia de Materiales de Aragón, CSIC-Universidad de Zaragoza, \\ Condensed Matter Physics Department, Zaragoza, Spain.
}

Case Study

Received $2^{\text {nd }}$ December 2011

Accepted $2^{\text {nd }}$ February 2012

Online Ready $4^{\text {th }}$ March 2012

\section{ABSTRACT}

Purpose: To clarify the origin of a metallic intraocular foreign body (IOFB) due to a firecracker injury and to show the utility of X-ray diffraction analysis in the identification process.

Methods: Interventional case report using X-ray diffraction technique.

Results: We report a mechanism of firecracker ocular injury not previously described in the published literature. It involved penetration into the eye by a ground particle of calamine (originated from the area of detonation) and not, as it could be suspected, by a firecracker fragment.

Conclusion: X-ray diffraction analysis showed environmental debris in the area of detonation can be injected as foreign material into the eye.

Keywords: Intraocular foreign body; firecracker injury; X-ray diffraction analysis; calamine; endophthalmitis; ultrasonography; computed tomography. 


\section{INTRODUCTION}

Work-related eye trauma is the major cause of ocular injury (Mansouri et al., 2009). The most common cause of intraocular foreign bodies (IOFBs) is still the hand-wielded hammer and chisel in male and young adults while working (Bai et al., 2010; Gupta A et al., 2010; Larque et al., 2010). X-ray diffraction technique is able to provide qualitative and quantitative information about the presence of certain compounds in an unknown mixture. In the present case this analysis demonstrated, according to the nature of the IOFB, that environmental debris in the area of detonation can be injected as foreign material into the eye, revealing a previously not described mechanism of firecracker injury. X-ray diffraction technique might be used to identify the nature of further intraocular small objects due to the easy use and wide availability.

\section{MATERIALS AND METHODS}

A 49-year-old man with an unremarkable medical history presented to the emergency department at the "Lozano Blesa" University Clinic Hospital, Zaragoza, Spain, two hours after being injured in his right eye by a firecracker. He was approaching to inspect a firecracker dud that initially failed to ignite when the explosion occurred. On examination, right eye presented a penetrating 1-mm corneosclerallimbal blast injury at 8 o'clock position with a concomitant iris laceration, traumatic cataract and a minimal vitreous hemorrhage. Visual acuity in the right eye was limited to the perception of hand motion and 20/30 in the uninjured left eye.

X-ray exam of the right orbit showed an almost imperceptible small radio-opaque foreign body (Figure $1 \mathrm{~A}$ ). Ultrasound examination (Figure $1 \mathrm{~B}$ ) and CT scan (Figure 1C) confirmed the presence of a metallic IOFB. Treatment with intravenous vancomycin (10 mg/kg/day divided q6hours) and ceftazidime (25 mg/Kg/day divided q8hours) was begun. After informed consent the patient was operated on the same day as he presented. Following primary wound closure, phacoemulsification of the cataract, scleral buckling and 20-gauge pars planavitrectomy (PPV) with posterior vitreous detachment were performed. After the PPV and clearing of the vitreous cavity with a backflush needle, a $0.7-\mathrm{mm}$ IOFB was visualized embedded in the inferotemporal retina. Argon laser photocoagulation at the site of impact of the foreign body was applied (Figure $1 \mathrm{D}$ ). Using intraocular forceps, the object was removed from the eye through the sclerotomy. A fluid-gas exchange with $25 \% \mathrm{SF}_{6}$ gas was done. An intravitreal injection of vancomycin $1 \mathrm{mg} / 0.1 \mathrm{~mL}$ and ceftazidime $2.25 \mathrm{mg} / 0.1$ $\mathrm{mL}$ was given. Postoperatively, the patient received for 1 week topical antibiotics (fluoroquinolone solution q2-3 hours) and corticosteroids (prednisolone $1 \%$ q2hr and then taper), as well as intravenous vancomycin (10 mg/kg/day divided q6hours), and his vision improved to 20/30 after just the first week. However, one week later, the right eye developed a posttraumatic endophthalmitis. Cultures of the vitreous aspirate were positive for Pseudomonas Aeruginosa and, although a new 20-gauge PPV was required, the patient lost finally his vision in this eye. 


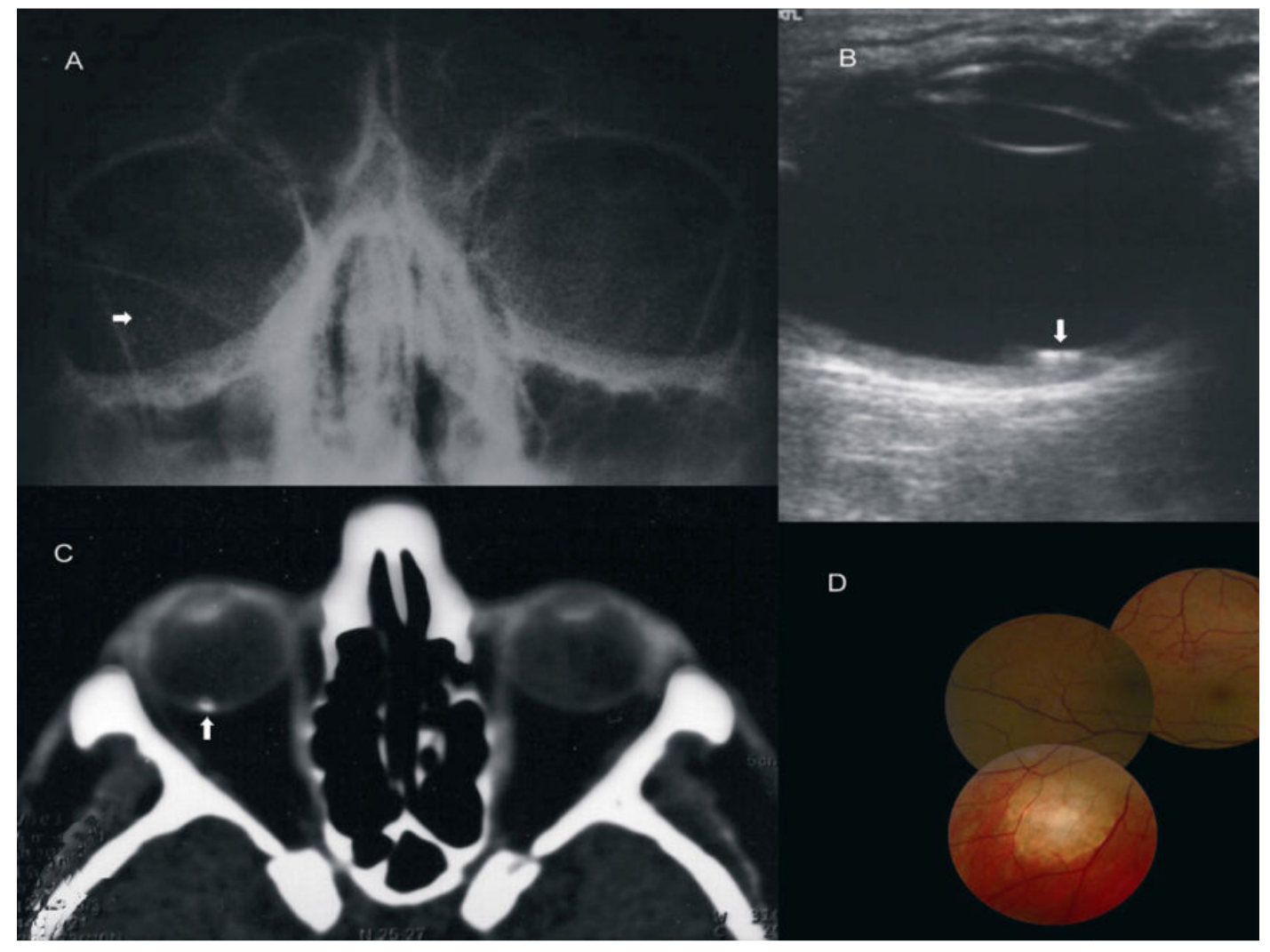

Figure 1. A: X-ray exam showed an almost imperceptible radio-opaque foreign body in the right orbit (arrow); B: Ultrasound examination clearly showing an intraocular foreign body embedded in the retina (arrow); C: CT scan confirmed the presence of a metallic intraocular foreign body in the right eye (arrow); D: Right fundus photograph after applying argon laser photocoagulation at the site of impact of the foreign body.

This research adhered to the tenetsof the Declaration of Helsinki and was approved by the Ethics Committee of our Institution. The foreign body was submitted to the Department of Condensed Matter Physics, University of Zaragoza, Spain, and analyzed by X-ray diffraction experiments using an Xcalibur $S$ diffractometer equipped with a flat Sapphire CCD area detector (manufactured by Oxford Instrumentation Ltd.). The small particle (inset B in Fig. 2) was pasted to the pin by means of a vacuum grease and the data were collected using Mo $\mathrm{K}_{\alpha}$ radiation $(\lambda=0.71073 \AA$ ) with an exposure time of $30 \mathrm{~min}$. Concentric Debye rings typical of a polycrystalline sample were created in the detector. Figure $2 \mathrm{~A}$ shows the right side of the rings. The scattered intensity was integrated in a small rectangular area from left to right in the middle of the Figure 2 using CrysAlis RED software (Oxford Diffraction Ltd., version 1.171.32.19). The resulting powder diffraction pattern after subtracting the background is plotted in the bottom inset $\mathrm{C}$ of Figure 2. 


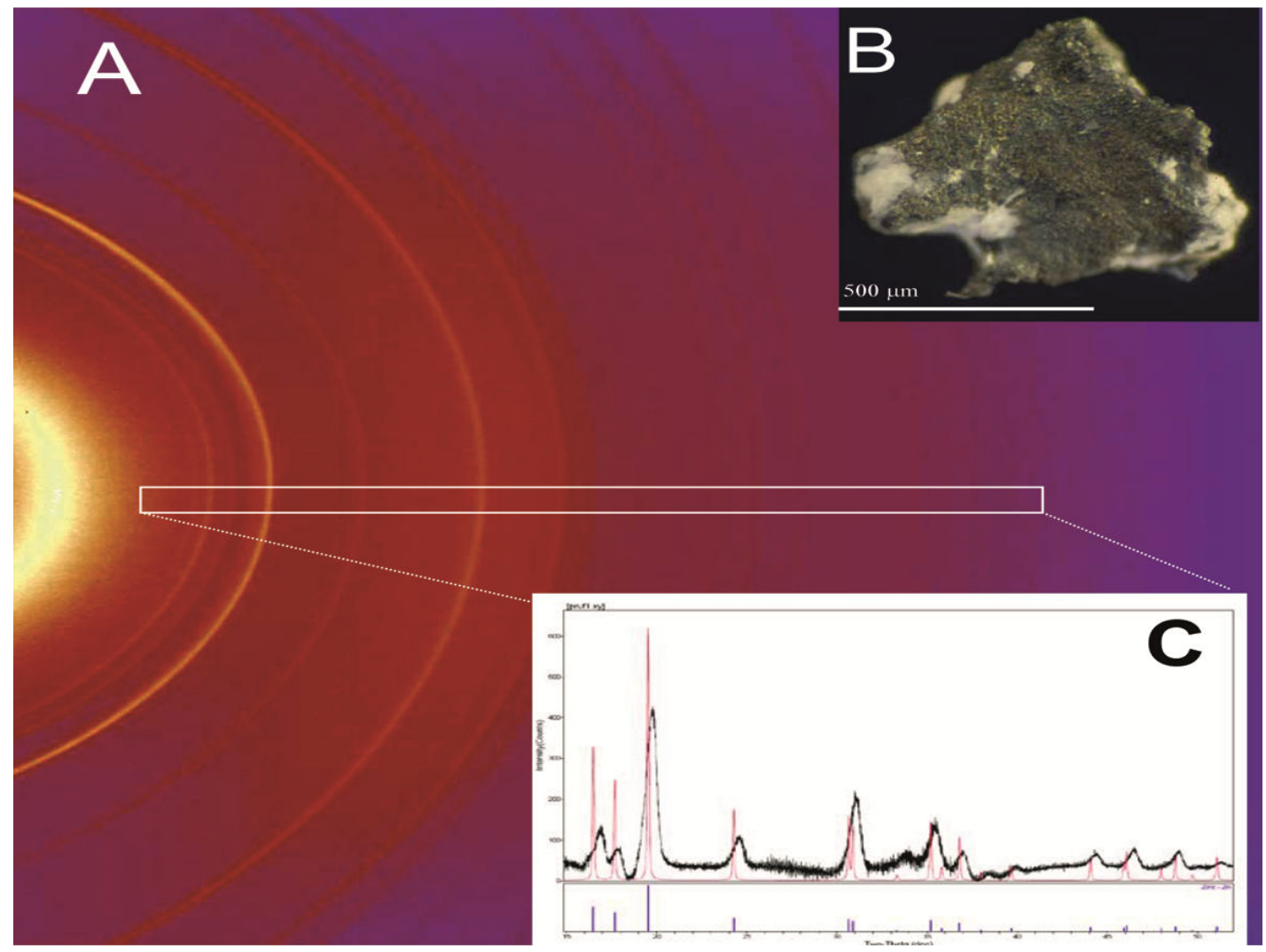

Figure 2. A: The x-ray diffraction pattern of the extracted particle obtained using Mo $K_{\square}$ radiation and recorded using a flat $C C D$ area detector which shows the concentric Debye rings typical of a polycrystalline sample. The white box delineates the area in which the scattered intensity was integrated (exaggerated for the sake of clarity). Inset B (top): A $0.7 \mathrm{~mm}$ calamine (melted $\mathrm{Zn}$ ) intraocular foreign body extracted from the eye through the sclerotomy. Inset $\mathrm{C}$ (bottom): Powder diffraction pattern obtained from the indicated rectangular area after subtracting the background. The lines at the bottom mark the theoretical position of the diffraction peaks corresponding to metallic Zn (card No. 87-0713 from ICDD). A theoretical pattern of a well crystallized $\mathrm{Zn}$ compound with sharp peaks is also included for the sake of comparison. This result reveals that the particle was formed by poor crystallized $\mathrm{Zn}$ metal.

\section{RESULTS \& DISCUSSION}

This pattern is composed of several diffractions peaks which are very broad. The broad peaks may arise from a poor crystallinity of the specimen. An automatic search-and-match was done along the powder diffraction file (PDF) database from ICDD (international centre for diffraction data, USA). One PDF record coincides with the experimental pattern including both peak positions and their intensities. It is also shown in the bottom inset $C$ of Figure 2 and it corresponds to $\mathrm{Zn}$ (card No. 87-0713). A theoretical pattern of a well crystallized $\mathrm{Zn}$ compound with sharp peaks is also included in the same figure. This result reveals that the particle was formed by poor crystallized $\mathrm{Zn}$ metal which might come from the firing of 
calamine ores or from melted $\mathrm{Zn}$. The presence of this kind of ores was visually confirmed in the gravel zone where the accident happened. Moreover, the composition of the firecracker did not include Zn.

X-ray diffraction technique is able to provide qualitative and quantitative information about the presence of certain compounds in an unknown mixture. Any crystalline material, which is made up of an ordered array of atoms, will give a diffraction pattern when it is interacting with $\mathrm{x}$-ray photons whose wavelength is similar to the interatomic distances. A diffraction pattern is characteristic of a particular atomic arrangement and therefore, it acts as a fingerprint of a particular compound.

We propose a new strategy for taking full advantage of this powerful analytical technique: the use of a diffractometer with kappa geometry widely used in the field of crystallography to structural resolution of small single crystals. This geometry allows the open access to the sample and its mounting on a pin holder with grease is uncomplicated. Collimation of the incoming beam ensures that only the sample is illuminated by the x-ray beam and the area detector collects Debye rings as in the old x-ray cameras (Figure 2). The integration of a rectangular area from the two dimensional pattern which is shown in this figure yields a conventional one dimensional diffraction pattern (inset of Figure 2). This pattern is ready to be used by a search-and-match program (Pecharsky and Zavalij, 2003).

Firecrackers (also known as crackers, noise makers, bangers or bungers) are small explosive devices primarily designed to produce a large amount of noise, especially in the form of a loud bang. They have fuses and are wrapped in a heavy paper casing to contain the explosive compound (Greene and Joholske, 2007). Detonation leads to a sudden release of energy dissipated by generation of heat, blast wave, and translocation of objects. The leading edge of a blast wave, which consists of few millimeters of over pressurized air, is called the blast front and it moves rapidly in all directions from the epicenter of the explosion. A blast wave can inflict primary blast biodynamic effects on the injured tissue. However, an explosion rapidly releases energy which provides a change in potential energy to kinetic energy in a very short period of time. So, the secondary blast effect is much more common than primary blast injury and plays a major role in ocular injuries from projectiles such as firecracker fragments, pebbles and sand particles with environmental debris in the area of detonation.

In our case, the x-ray diffraction identified the IOFB as metallic zinc (Zn). Zn has a limited use in firecrackers. The patient experienced the accident with a small explosive containing black powder in a tight paper tube. The classical black powder contains charcoal, sulfur and potassium nitrate. Sometimes aluminum powder is added to charcoal in order to brighten the explosion. $\mathrm{Zn}$ is not present in the chemical composition of these small crackers devoted to produce a maximum audio report (Donner, 1997). Accordingly, IOFB should arise from the explosion vicinity. The patient confirmed that the accident happened in a soil area with calamine remainders coming from a factory devoted to the galvanization of iron tools. The main limitation of the study was that no samples of the gravel zone were analyzed by $x$-ray diffraction analysis. Another limitation is that x-ray diffraction technique applied might only be applied to crystalline compounds mainly and no other materials.

High energy explosions can inject foreignmaterial into the eye, resulting in an IOFB, which may cause devastating damage to the eye. The clinical history is important, and details regarding the location where injury occurred (i.e., home, construction site, gravel) should be obtained, because penetrating fragments range from paper casing to ground particles. The 
occurrence of casualties involving the eye and orbit isto some extent dependent on the terrainin which it is exploded. The patterns of ocular injuries are also a result of the composition and type of explosive and carrier/container delivery method, the distance between the victim and the blast epicenter, whether the blast occurred in a closed or open space, and any surrounding environmental barriers or hazards.

IOFB rarely cause infection, perhaps because of their heat and speed. However, with an IOFB resulting from an explosion, as well as in trauma involving vegetable matter in rural settings, the possibility of infection is up to 30\% (Al-Mezaine et al., 2010). In these cases, bacteria such as Bacillus cereus, Staphylococcus aureus, Streptococcus pneumoniae, and Pseudomonas aeruginosa can lead to extremely poor visual results (Lieb et al., 2003; AlQmran et al., 2007). In this case, the knowledge about the composition and origin of IOFB obtained by $x$-ray diffraction implied a worse prognosis of the accident.

Understanding the pathophysiology of blast injuries to the retina and vitreous as well as the new vitreoretinal surgical techniques will help in the management of traumatized eyes with blast wave and penetrating foreign body. Nevertheless, despite advancements, the prognosis remains guarded for any eye with an IOFB (Yang et al., 2010). Preventive safety measures when working with firecrackers, such as wearing safety glasses, have been shown to be effective in reducing the number of injuries (Sundelin and Norrsell, 2000).

\section{CONCLUSION}

In the present case, X-ray diffraction analysis contributed to identify the nature of an IOFB, demonstrating that environmental debris in the area of detonation can be injected as foreign material into the eye. This mechanism of firecracker ocular injury, has not been previously reported in the literature, It consists in the penetration into the eye by a ground particle of calamine (originated from the area of detonation) and not by a firecracker fragment (which did not include $\mathrm{Zn}$ ). This technique can be used to identify further crystalline intraocular small objects due to the easy use and wide availability.

\section{ACKNOWLEDGEMENTS}

The authors acknowledge the facility of the Servicio de Difracción de rayos $X$ in the Zaragoza University to acquire diffraction patterns.

\section{COMPETING INTERESTS}

Authors have declared that no competing interests exist.

\section{REFERENCES}

Al-Mezaine, H.S., Osman, E.A., Kangave, D., Abu El-Asrar, A.M. (2010). Risk factors for culture-positive endophthalmitis after repair of open globe injuries. Eur. J. Ophthalmol. 20, 201-208.

Al-Omran, A.M., Abboud, E.B., Abu El-Asrar, A.M. (2007). Microbiologic spectrum and visual outcome of posttraumatic endophthalmitis. Retina, 27, 236-242. 
Bai, H.Q., Yao, L., Meng, X.X., Wang, Y.X., Wang, D.B. (2011). Visual outcome following intraocular foreign bodies: a retrospective review of 5-year clinical experience. Eur. J. Ophthalmol, 21, 98-103.

Donner, J. (1997). A professional's guide to pyrotechnics, Boulder, CO: Paladin Press Ed., pp. 21-28.

Gartanganis, S.P., Kanellopoulou, D.G., Mela, E.K., Panteli, V.S., Koutsoukos, P.G. (2008). Opacification of hydrophilic acrylic intraocular lens attributable to calcification: Investigation on mechanism. Am. J. Ophthalmol, 146, 395-403.

Goll, J.G., Wilkinson, L.J., Snell, D.M. (2009). Teaching chemistry using October sky. J. Chem. Edu., 86, 177-180.

Greene, M.A., Joholske, J. (2007). 2006 Fireworks Annual Report: Fireworks-Related Deaths, Emergency Department Treated Injuries, and Enforcement Activities During 2006, Washington (DC): U.S. Consumer Product Safety Commission.

Gupta, A., Srinivasan, R., Babu, K.R., Setia, S. (2010). Comparison of the clinical presentation and visual outcome in open globe injuries in adults and children over 30 months. Eur. J. Ophthalmol, 20, 590-595.

Hardt, A. (2001). Pyrotechnics. Post Falls, Idaho: Pyrothechnica Pubs, USA, Ch 15.

Jenkins, R. (1999). X-ray fluorescence spectrometry, $2^{\text {nd }}$ ed, New York: John Wiley \& Sons, Ch. 4.

Kleinmann, G., Apple, D.J., Werner, L., Pandey, S.K., Neuhann, I.M., Assia, E.I., et al. (2006). Postoperative surface deposits on intraocular lenses in children. J. Cataract. Refract. Surg., 32, 1932-1937.

Lai, J.Y., Chen, K.H, Hsu, W.M., Lee, T.H., Lin, S.Y. (2008). Multiple elements in the deposits of opacifiedhydroview Intraocular Lens. Am. J. Ophthalmol. 139, 1123-1125.

Larque, A.B., Peralta, J., Lopez, J. (2010). Epidemiology of open-globe trauma in the southeast of Spain. Eur. J. Ophthalmol, 20, 578-583.

Lieb, D.F., Scott, I.U., Flynn, H.W. Jr., et al. (2003). Open globe injuries with positive intraocular cultures: factors influencing visual acuity outcomes. Ophthalmology, 110, 1560-1566.

Mansouri, M.R., Hosseini, M., Mohebi, M., Alipour, F., Mehrdad, R.(2009). Work-related eye injury: the main cause of ocular trauma in Iran. Eur. J. Ophthalmol, 20, 770-775.

Schrago, N.F., Reim, M., Burchard, W.G., Teping, C., Wenzel, M. (1992).Scanning electronmicroscopic and energy-dispersive $\mathrm{x}$-ray-analysis findings on 2 brand new intraocular lenses. Ophthalmic, Res., 24, 51-54.

Sundelin, K., Norrsell, K. (2000). Eye injuries from fireworks in Western Sweden. Acta. Ophthalmol, 78, 61-64.

Welch, R. (1975). Two remarkable events in the field of intraocular foreign body: (1) the reversal of siderosisbulbi (2) the spontaneous extrusion of an intraocular copper foreign body. Trans. Am. Ophthal. Soc., 73, 187-203.

Yang, C.S., Lu, C.K., Lee, F.L., Hsu, W.M., Lee, Y.F., Lee, S.M. (2010). Treatment and outcome of traumatic endophthalmitis in open globe injury with retained intraocular foreign body. Ophthalmologica, 224, 79-85.

(C) 2012 Ascaso \& Blasco; This is an Open Access article distributed under the terms of the Creative Commons Attribution License (http://creativecommons.org/licenses/by/3.0), which permits unrestricted use, distribution, and reproduction in any medium, provided the original work is properly cited. 